\title{
Electromagnetic Scattering by Gyrotropic Cylinders With Axial Magnetic Fields ${ }^{1}$
}

\author{
W. C. Y. Lee, ${ }^{2}$ Leon Peters, Jr., and C. H. Walter
Contribution From the Antenna Laboratory, Department of Electrical Engineering, Ohio State University, Columbus, Ohio

(Received September 3, 1964;

\begin{abstract}
The modified geometrical optics method is applied to find the radar cross section of an infinite, gyrotropic, circular cylinder with an axial magnetic field. The results compare favorably with those obtained using the boundary value solution. The modified geometrical optics method offers the advantage that it may be applied to a general cylindrical cross section without further development. It may also be applied to other orientations of magnetic field but the computations are much more tedious.
\end{abstract}

\section{Introduction}

The modified geometrical optics method [Peters and Thomas, 1962; Thomas, 1962; Kawano and Peters, 1963; Kouyoumjian, Peters, and Thomas, 1963; Kawano and Peters, 1964] has been successfully applied to the dielectric body and the plasma body to obtain their radar cross sections. This modified geometrical optics method is applied in this paper to obtain the radar cross section of a gyrotropic cylinder with an axial magnetic field. The only previously existing solution for the radar cross section of gyrotropic bodies is one obtained by Wait [1961] for the infinite plasma cylinder with the static magnetic field alined parallel to the cylinder axis. There is little hope that boundary value solutions may be obtained for other anisotropic bodies. On the other hand, there is no theoretical limitation on the applicability of the modified geometrical optics method to such scattering problems. There is only the practical limitation that the solution may be quite lengthy and difficult in certain cases.

The purpose of this paper is to compare the radar cross section obtained using the boundary value solution of Wait and the modified geometrical optics method to further demonstrate the capabilities of this latter method. The modified geometrical optics method has already been applied to a body for which no other solution is available [Lee, Peters, and Walter, 1964a and b].

It may be noted that a cylindrical plasma region in many cases represents a practical configuration. Some examples are the satellite trail in the ionosphere, meteor trails, and an ionized shock wave from an exploding wire.

1 The work reported in this paper was supported in part by Contract Number AF 33(616)-7270 between Air Force Cambridge Research Laboratories, Office of Aerospace Research, Bedford, Mass., and The Ohio State University Research Foundation.

2 Presently employed by Bell Telephone Laboratories, Inc.

\section{Geometry of the Gyrotropic Cylinder}

The reflected or scattered field of a cylindrical plasma is derived under the assumption that the ionized column is infinite in length and the column is assumed to be homogeneous. A cylindrical column with static magnetic field $\bar{Y}$ directed along the negative $y$-axis is shown in figure 1 , where

$$
\begin{aligned}
\bar{Y} & =\frac{\bar{\omega}_{H}}{\omega}=-\frac{\mu_{0} e \bar{H}_{0}}{m \omega}=Y_{y} \hat{i}_{y} ; \\
\mu_{0} & =\text { permeability, } \\
e & =\text { charge of electron, } \\
\bar{H}_{0} & =H_{0} \hat{i_{y}}=\text { applied static magnetic field, } \\
m & =\text { mass of electron, and } \\
\omega & =2 \pi f .
\end{aligned}
$$

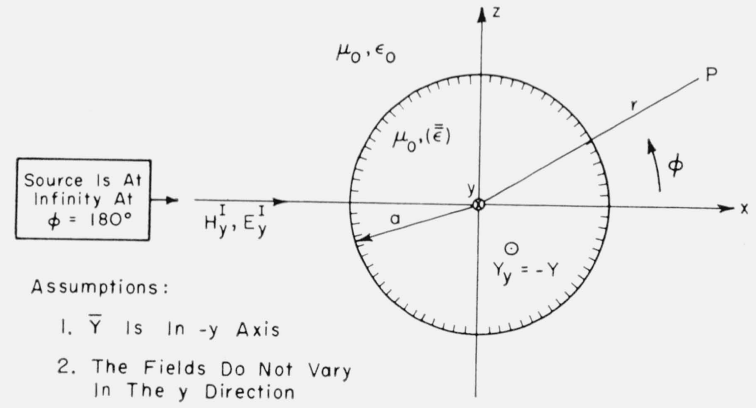

FIGURE 1. A cylindrical column with static magnetic field $\overline{\mathrm{Y}}$ oriented along negative y axis. 
The fields $H_{y}$ and $E_{y}$ individually satisfy the wave equations, and this decomposition is into independent partial waves. The solution for the case $H_{y}=0$ is relatively trivial (since the constant magnetic field $Y_{y}$ has no influence) and is known as the ordinary wave. This solution can be obtained by using the regular modified geometrical optics method. The wave associated with $E_{y}=0$ is known as the extraordinary wave and is the case considered in this paper.

When a plane wave is incident on the plasma cylinder of figure 1 , with $E_{y}=0$, there is only one extraordinary ray emanating into the cylinder at each point of incidence on the cylinder. Further, the index of refraction associated with each of these extraordinary rays is not dependent on the point of incidence or the direction of the ray path and is given by the Appleton-Hartree equation. Thus in applying the modified geometrical optics method, there is little change from the methods developed for the isotropic cylinder. In fact, the only significant change is in the various reflection and transmission coefficients which are dependent upon the point of incidence. This presents no real difficulty when the modified geometrical optics method is applied but requires that the boundary value solution must be solved anew, as has been done by Wait [1961].

\section{Modified Geometrical Optics}

The modified geometrical optics method as developed by Kouyoumjian et al. [1963], represents an extension of the more commonly used geometrical optics. Optical methods are used with the modification that physical optics is applied to determine the magnitude of the scattered field where geometrical optics techniques fail, i.e., physical optics is used to find a value for the spatial attenuation factor. This method has been highly successful for determining: the radar cross section of plasma and dielectric bodies.

The first step in application of the modified geometrical optics method is to determine the significant ray paths. These are the rays that are pointed in the vicinity of the direction for which the scattered field is to be computed. These various rays are classified in terms of the number of internal reflections that they undergo. They are further subdivided in terms of the glory ray and stationary ray which are to be discussed later.

A field $U(l)$ associated with a ray is described in a general manner by

$$
U(l)=A e^{-j \phi} F(l) e^{-j k l},
$$

where

$A$ is the reference amplitude,

$\phi \quad$ is the reference phase,

$F(l)$ is the spatial attenuation factor, and $e^{-j k l}$ is the spatial phase-delay factor.

The ray field is given by (2) and the spatial attenuation, $F(l)$, is of primary interest. When geometrical optics is applicable, $F(l)$ is readily obtained by the conservation of energy. When ordinary geometrical optics is not applicable, $F(l)$ can be obtained by means of physical optics. Two such applications which have been treated in detail are the glory ray and the stationary ray.

The solutions for $F(l)$ for $m=1,2,3$ internal reflections are tabulated in table 1 for the glory ray contribution.

TABle 1. Parameters of internal reflections for $m \leq 3$

\begin{tabular}{|c|c|c|c|}
\hline & $m=1$ & $m=2$ & $m=3$ \\
\hline$\frac{\Delta \theta_{0}}{\Delta \gamma}$ & $-1+4 \frac{d \theta_{1}}{d \theta_{0}}$ & $-1+6 \frac{d \theta_{1}}{d \theta_{0}}$ & $-1+8 \frac{d \theta_{1}}{d \theta_{0}}$ \\
\hline
\end{tabular}

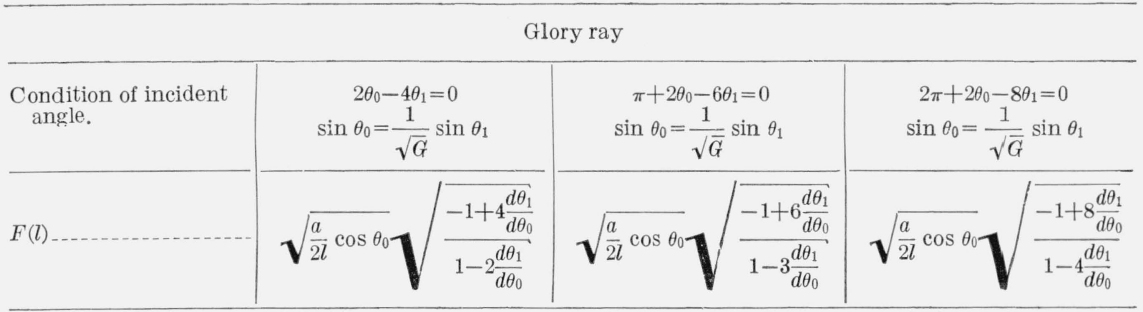

Stationary ray

\begin{tabular}{l|c|c|c}
\hline $\begin{array}{l}\text { Condition of incident } \\
\text { angle. }\end{array}$ & $\cos \theta_{0}=\sqrt{\frac{1-G}{3 G}}$ & $\cos \theta_{0}=\sqrt{\frac{1-G}{8 G}}$ & $\cos \theta_{0}=\sqrt{\frac{1-G}{15 G}}$ \\
\hline $\cos \theta_{1}=2 \sqrt{\frac{1-G}{3}}$ & $\frac{3}{\cos \theta_{1}=3 \sqrt{\frac{1-G}{8}}}$ & $\frac{\cos \theta_{1}=4 \sqrt{\frac{1-G}{15}}}{9 \cot \theta_{s} \cdot \cos ^{2} \theta_{s}}$ & $\frac{15}{16 \cot \theta_{s} \cdot \cos ^{2} \theta_{s}}$ \\
\hline
\end{tabular}


Definition of symbols of table 1:

$\theta_{0}$ is the angle of incident ray with respect to the normal to the surface,

$\theta_{1}$ is the angle of the refracted ray with respect to the normal,

$m=$ number of internal reflections,

$G=\frac{1}{n^{2}}=\frac{1-X-Y^{2}}{(1-X)^{2}-Y}$,

$n=$ index of refraction,

$X, Y$ are the usual plasma parameters.

The spatial attenuation factor for the stationary ray is given by

$$
F(l)=\frac{s}{\lambda l} 2\left|A_{i}(z)\right|
$$
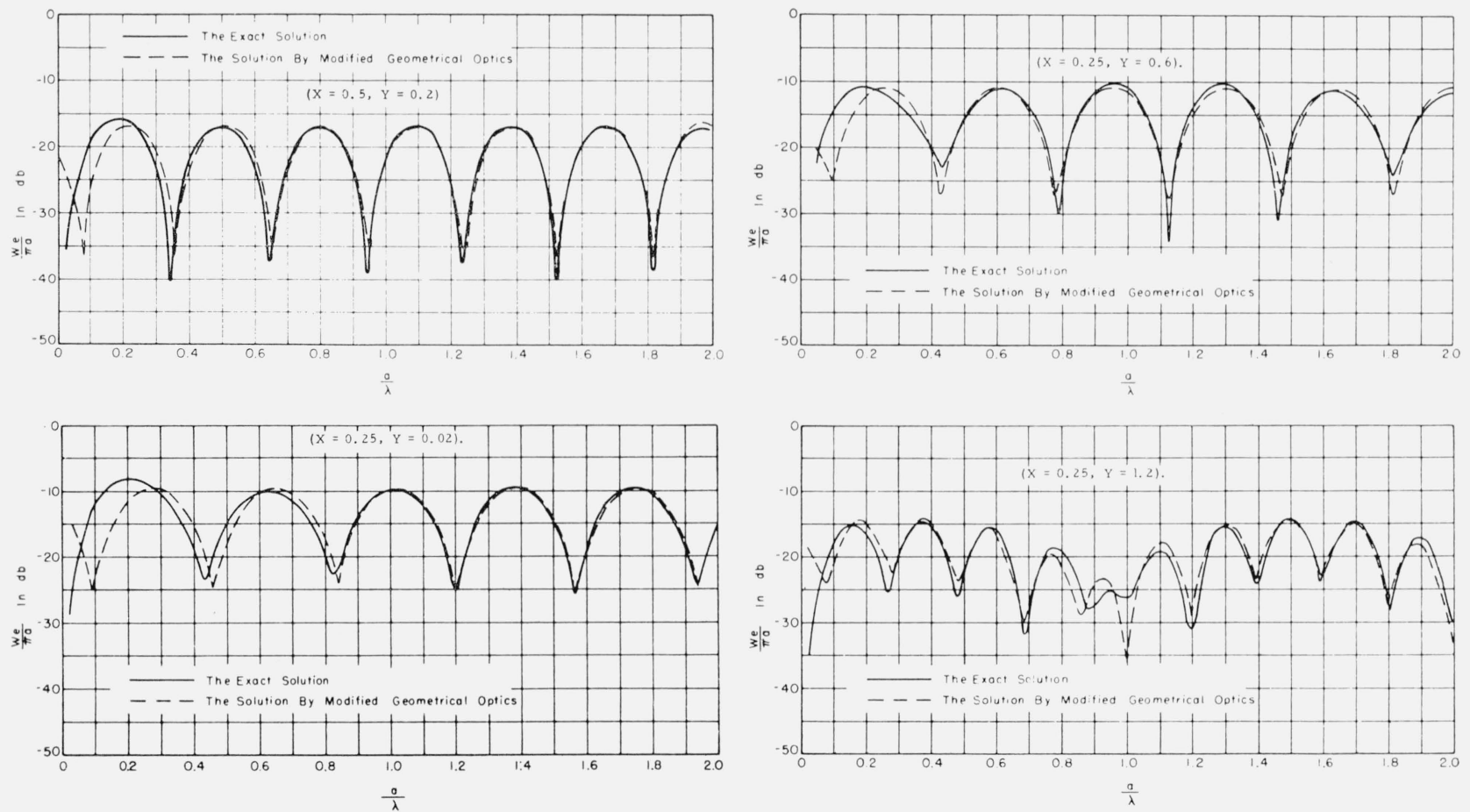

where

$s=\left(\frac{a^{2}}{k h}\right)^{1 / 2}$

$z=-k s \xi_{s}$,

$\xi_{s}=$ is the exit angle for the stationary ray,

$\xi=-2 \pi+2 \theta_{0}+(m+1)\left(\pi-2 \theta_{1}\right)$

$=$ exit angle,

$\theta_{s}=$ value of $\theta_{0}$ for the stationary ray,

$h$ is given in table 1 , and

$A_{i}$ is the Airy integral.

The phase of each contribution to the radar cross section must be carefully preserved. Attention must be given to the phase jumps that occur as the ray passes through a caustic and the total electrical path length must be computed. The detailed

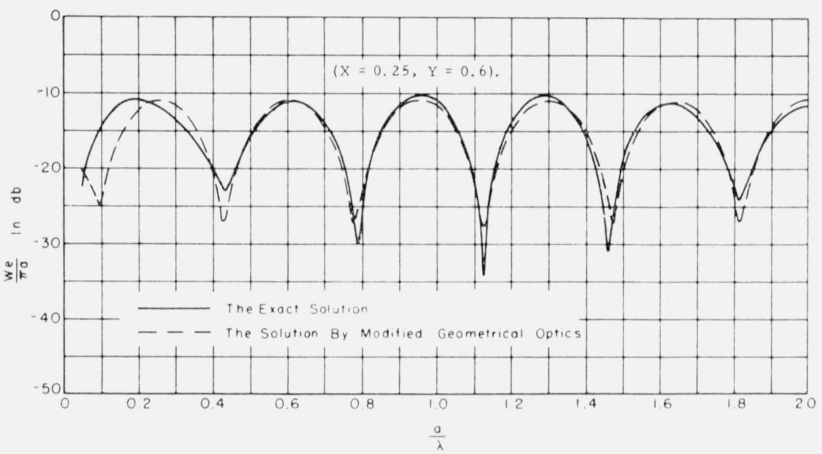

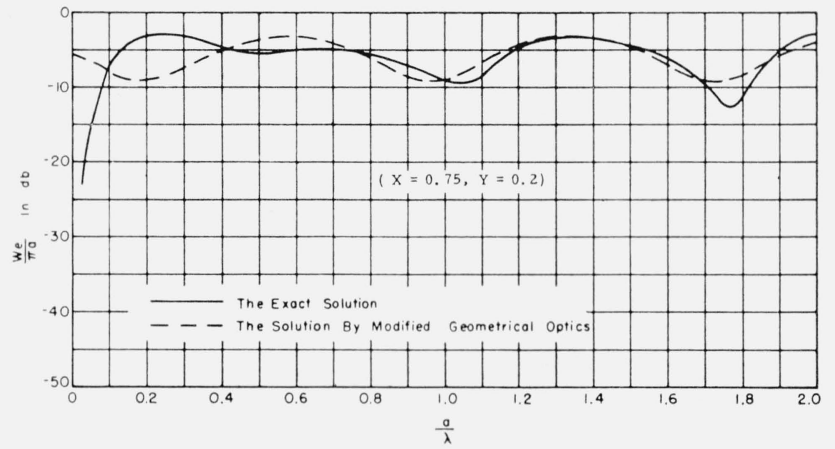

FIGURE 2. Comparison of exact solution [Wait, 1964] and approximate backscatter echo width of an infinite circular cylinder with a static magnetic field oriented along the cylinder axis. 
procedure for this computation has been given in a previous paper [Kawano and Peters, 1963]. The radar cross section is readily obtained from the phasor sum of the scattered fields as computed by the modified geometrical optics method.

\section{Numerical Results}

These methods have been applied to several typical cases and the results compare favorably with those calculated from Wait's [1961] boundary value solution as shown in figure 2 . The results obtained from the boundary value solution a re the only known numerical values.

\section{Conclusions}

The modified geometrical optics method has been applied to find the radar cross sections of a number of homogeneous gyrotropic cylinders with axial magnetic fields. The results obtained using this method compare favorably with those obtained using the boundary value solution.

This particular case is quite similar to the usual isotropic cylinder in that the index of refraction is independent of the direction of propagation. It is this fact that makes the boundary value solution possible. No similar boundary value solution has been obtained for any other orientations of magnetic field.

The only new requirement for the application of modified geometrical optics consists of introducing different reflection and transmission coefficients. It can be applied to any orientation of magnetic field. Several geometries have been considered in another paper [Lee, Peters, and Walter, 1964a and b].

The present application is of value for several reasons. It demonstrates further the versatility of this modified geometrical optics method and lends additional confidence to its application to more complex problems such as the gyrotropic body with an arbitrarily oriented magnetic field. No new concepts are needed to treat the general cylindrical gyrotropic body with axial magnetic field.

Finally, the computations made in the present study are far easier to make using the modified geometrical optics method than those using the boundary value solution.

\section{References}

Kawano, T., and L. Peters, Jr. (1963), An application of modified geometrical optics method for bistatic radar cross section of dielectric bodies, Antenna Laboratory, The Ohio State University Research Foundation, 1116-39.

Kawano, T., and L. Peters, Jr. (1964), An extension of the modified geometrical optics methods for radar cross sections of dielectric bodies, Antenna Laboratory, The Ohio State University Research Foundation, 1116-38.

Kouvoumjian, R. G., L. Peters, Jr., and D. T. Thomas (1963), Modified geometrical optics method for scattering by dielectric bodies, IEEE Trans. Ant. Prop. AP-11, No. $690-703$.

Lee, W. C. Y., L. Peters, Jr., and C. H. Walter (1964a), Modified geometrical optics for electromagnetic scattering by gyrotropic bodies, Antenna Laboratory, The Ohio State University Research Foundation, 1116-47.

Lee, W. C. Y., L. Peters, Jr., and C. H. Walter (1964b), Geometrical optics for gyrotropic bodies, Antenna Laboratory, The Ohio State University Research Foundation, 1116-45.

Peters, L., Jr., and D. T. Thomas (1962), A geometrical optics approach for the radar cross section of thin shells. J. Geo-phys. Res. 67, 2073.

Thomas, D. T. (1962) Scattering by plasma and dielectric bodies, Antenna Laboratory, The Ohio State University Research Foundation, 1116-20.

Wait, J. R. (April-June 1961), Some boundary value problems involving plasma media, J. Res. NBS 65B (Math. and Math. Phys.) No. 2, 137-150.

(Paper 69D-457) 